\title{
Desarrollo de habilidades cognoscitivas y de pensamiento crítico desde una problemática ambiental
}

\author{
Development of cognitive skills and of critical thought from an environmental \\ problematics
}

Yesica Danisa Carvajal Alfonso y Tatiana Alejandra Huertas Navarro

Estudiantes de Licenciatura en Química. Universidad Pedagógica Nacional. yesidani@hotmail.com , talehuna@hotmail.com

\section{Resumen}

En las ultimas décadas se han visto diversos e interesantes cambios en la forma como se dan los procesos de enseñanza y aprendizaje en la escuela y en aula (Bermúdez, Escobedo \& Jaramillo, 2.002), razón por la cual se planea un análisis teórico reflexivo en el cual se proponen algunas posibles herramientas didácticas para generar habilidades cognoscitivas que fomenten y fortalezcan el pensamiento critico y reflexivo en los estudiantes frente a la química tomando como punto de referencia aspectos de la problemática ambiental que se está viviendo actualmente, y la importancia que está generando la producción e implementación de combustibles alternativos debido a la necesidad de lograr un desarrollo sustentable y menos contaminante del medio ambiente.

\section{Palabras clave}

Habilidades Cognoscitivas y de pensamiento critico y reflexivo, combustibles alternativos, software educativo.

\section{Abstract}

In the last decades diverse and interesting changes have been seen in the form like give the processes of education and learning in the school and in dassroom (Bermúdez, Escobedo and Jaramillo, 2.002), reason by which there is planned a theoretical reflexive(reflective) analysis in which they propose some possible didactic tools to generate cognitive skills that foment and strengthen the critical and reflexive thought in the students opposite to the chemistry taking as a point of reference aspects of the environmental problematics that is lived nowadays, and the importance that is generating the production and implementation of alternative fuels due to the need to achieve a development sustentable and less pollutant of the environment.

\section{Keywords}

Cognitive skills and of thought criticize and reflexively, alternative fuels, educational software.

\section{Introducción}

Las exigencias tanto sociales como culturales de un país en vía de desarrollo, como lo es Colombia, plantean la necesidad de crear nuevas estrategias y/o metodologías que contribuyan a mejorar los procesos de enseñanza - aprendizaje de la química en el aula de clase. De igual manera dentro de los grandes cambios que ha sufiido en las ultimas décadas la humanidad se ha observado el impresionante desarnollo de la ciencia y la tecnología (Salcedo, Villareal, Zapata, Colmenares, Garća \& Romero, 2008) modificando así la forma de comunicación entre las personas 
con la implementación masiva de nuevas herramientas virtuales y tecnológicas en el aula de dase. Debido a esto se plantea la necesidad de desarrollar una herramienta didáctica que permita tomar las TICs (Tecnologías de la información y la comunicación) como un instrumento que permita potencializar la relación estudiante - conocimiento, y poder generar en él, habilidades de pensamiento critico y refiexivo ya que las demandas actuales de la educación así lo establecen. Sin embargo, existe actualmente otra situación no menos relevante que esta modificando no solo la sociedad Colombiana sino el mundo entero, ya que los cambios ambientales que se viven diariamente no pueden pasar desapercibidos frente a los ojos de nadie debido a los daños que están causando en el planeta. Debido a esto se han buscado nuevas altemativas que contribuyan al no deterioro del medio ambiente, razón por la cual programas como el de Naciones Unidas para el Medio Ambiente (PNUMA) han sido creados con el objetivo de promover la cooperación internacional en cuanto a medio ambiente se refiere; además, se ha comenzado a desarrollar fuentes de energía alternativas, las cuales no generen tantos daños en la tienra y contribuyan de esta manera a su preservación, una de estas es el Biodiesel.

El fundamento teónico de este proyecto se enmarca en los siguientes aspectos:

\section{Competencias Cognoscitivas y Científicas}

Desde los años Setenta en Colombia, las nuevas expectativas sociales exigían que el sistema educativo fuera más allá de garantizar escolaridad universal, que ofreciera a todos los estudiantes, oportunidades para desarrollar las habilidades y valores necesarios para vivir, convivir, ser productivo y seguir aprendiendo a lo largo de la vida. Comenzó, entonces a ser relevantes diversos conceptos que se engloban como componentes importantes para conseguir llegar a una educación de Calidad, "Las Competencias Científicas, junto con la evaluación y los Planes de Mejoramiento son una de esas herramientas en la cual viene trabajando el Ministerio de Educación desde 2002 " (Vélez, 2007).

Naturalmente al pensar que una Educación de Calidad, comprende y acopla en su estructura una Enseñanza-Aprendizaje por Competencias, necesariamente plantea la necesidad de forjar un modelo de formación por Competencias, pero ese no ha sido el caso colombiano, lo que ha obligado a académicos y pedagogos a repensar la educación de los estudiantes bajo un modelo de formación por Competencias. Formular, Validar y Socializar dichas Competencias, se han constituido en un trabajo exigente y riguroso que consulta el saber pedagógico, la práctica escolar, la innovación e investigación educativa y pedagógica, la evaluación, el avance del conocimiento disciplinar y didáctico.

Pero ¿Qué son las Competencias en el contexto Educativo?, son diversos los discursos teóricos que se han dado sobre los diferentes estadios de la historia, las más actuales por ejemplo son las otorgadas por el Instituto Colombiano para el Fomento de la Educación Superior -ICFES (1999) y el Ministerio de Educación Nacional (2008), en donde afirman que las Competencias son un saber hacer en contexto que requieren la aplicación creativa, flexible y responsable de conocimientos, habilidades y actitudes. De igual manera aparecen definiciones más antiguas y de carácter Psicológicas como (Torrado, 1999), Comunicativo (Hymes, 1972), Laborales (Delors ,1996;Gómez, 1998), conceptos que dejan atrás posiciones Conductistas y Empiropositivistas (como la tomada por Skinner) posiciones Lingüísticas como la que sostiene Chomsky (1971) al definir la competencia como el dominio de los principios que gobieman el lenguaje; y la actuación como la manifestación de las reglas que subyacen al uso del lenguaje, Un enfoque similar es el de Piaget, que con ayuda de elaboraciones epistemológicas Constructivistas postula que esas reglas y principios están subordinadas a una lógica de funcionamiento particular, y no a una lógica de funcionamiento común. Por su parte Vigotsky propone que el desarrollo cognitivo, mas que derivarse del despliegue de mecanismos internos, resulta del impacto que tiene la cultura sobre el 
individuo en la realización de las funciones sicológicas, como en el caso del lenguaje. Por ello la competencia puede entenderse como "capacidad de realización, situada y afectada por y en el contexto en que se desenvuelve el sujeto".

\section{Importancia de desarrollar competencias cognoscitivas, como} preámbulo para el desarrollo de competencias científicas

En la Actualidad, se ha dado inicio a la formulación de Proyectos curriculares, con especial énfasis en la construcción de Competencias Cognoscitivas. Gallego Badillo, R. y Pérez Miranda, R, (1998), antes de adentrar a los estudiantes para que desarrollen competencias científicas, siendo imprescindible las Teorías psicológicas sobre el Aprendizaje, en donde se requiere discutir las ideas de Inteligencia, Actitud, Aptitud y Contenido, que en un sentido estricto se convergen dando lugar a los llamados Aprendizajes significativos, que son los aprendizajes en los cuales el sujeto del proceso de formación reconfigura la información nueva con la experiencia, permitiéndole así integrar grandes cuerpos de conocimiento con sentido. De esa integración entre conocimiento con sentido y experiencia resulta el desarrollo de la competencia.

El calificativo Cognoscitivo que se le da a las Competencias, según Gallego Badillo, $\mathbf{R}$ y Pérez Miranda, R, (1998) es una actividad de discusión epistemológica, Pedagógica y didáctica, que permite que dichas competencias se endaven en una dinámica no lineal, que le dan el sentido de la creatividad, son construcciones de cada ser humano en comunidad, en el interior de escenarios preparados para tal efecto. Es así, por cuanto se admite que cada estudiante, desde su inmersión en el saber común y cotidiano tanto como a partir de sus experiencias de formación en el sistema educativo institucional, ha de haber elaborado unas competencias, que deben ser objeto de trabajo pedagógico y didáctico para sus correspondientes reconstrucciones. En el intento de explicitación de proponer unas Competencias Cognoscitivas, y apoyándose en Piaget, (1983), tanto como en el problema de las actitudes Gallego Badillo, R. y Pérez Miranda, R, (1999), se llega a la condusión que esas Competencias se desarrollan en tres dimensiones; La Cognoscitiva: Porque la construcción de Competencias se da en el interior de un saber determinado, en la medida que se estructura conceptual y metodológicamente, La Actitudinal y la Actuacional: Realizaciones o desempeños concretos en escenarios espeáficos. Estas tres dimensiones adquieren su fundamentación en la relación holística que hace que cada una de ellas no pueda ser comprendida sin las otras. A la vez cada una de estas dimensiones, para efectos pedagógicos y didácticos, se ha especificado en unas subdimensiones: Conceptual, Metodológica, estética, Actitudinal y Axiológica. Se afirma que solo después de que se ha llegado a la comprensión, de que las competencias tienen su base y fundamentación en la actividad cognitiva, se le podrá dar un sentido Cientúfico a dichas Competencias.

En el caso particular de la educación en Colombia, y teniendo en auenta Investigaciones Históricas, Epistemológicas, Pedagógicas Y Didácticas, como las realizadas por (Ibáñez, X et al, 2006), en donde se han querido promover, en especial, Competencias Científicas dentro del aula, traduciéndose espeáficamente en "Competencias y Desempeños orientados por los Profesores y observables en los estudiantes", se categorizan las Competencias como Básicas, Investigativas y de Pensamiento Crítico, en niveles inicial, Intermedio y avanzado, en especial y para la investigación que corresponde a este Proyecto de grado se hace especial énfasis en las Competencias Cient́ficas de Pensamiento crítico y Reflexivo, que entre otras tienen características importantes como:

Las Competencias Científicas de Pensamiento Crítico y Reflexivo se entienden como la capacidad que tiene un sujeto de desarrollar procesos cognitivos que van más allá de la selección y procesamiento de la información, permitiéndole integrar creativa y prepositivamente los saberes frente a nuevas situaciones, resolviendo problemas desde una postura cótica, ética y de construcción de significados contextualizados. Dicha postura crítica implica, que además de comprender los fenómenos, se construya una mirada particular en tomo a la ciencia misma y sus 
implicaciones en la sociedad, reconociéndose como un sujeto dentro del mundo natural y social. La construcción de significados está ligada al reconocimiento, contrastación e interpretación que hace el sujeto de diversas fuentes de información, elaborando su propio universo de comprensión sobre los eventos y fenómenos naturales Cárdenas, F. Sarmiento, F. (2000).

\section{Los combustibles renovables}

La enseñanza de la química actualmente supone relacionar conceptos básicos, generalmente abstractos, con situaciones de la vida cotidiana (Santana, Navarro, Femández, González y Domínguez, 2005), ya que los problemas que presenta el planeta tierra se hacen cada vez mas notorios, tal es el caso del "calentamiento de la atmósfera ayyo principal desafío medioambiental que hoy afronta la humanidad a nivel mundial" es encontrar la forma de disminuir la producción de los gases responsables del fenómeno llamado "Efecto Invemadero" causados por el uso de combustibles fósiles (petróleo y carbón) como fuente de energía. Diversas investigaciones se han dado con el propósito de generar combustibles alternativos como fuente de energía para contrarrestar el efecto de los combustibles que actualmente se están utilizando, una de las posibles opciones es conocido como "Biodiesel".

Se entiende como biodiesel al éster metílico producido a partir de los aceites vegetales y grasas animales, siendo la colza, el girasol y la soja las materias primas más utilizadas para su uso como biocarburante, donde biocarburante se define como combustible líquido o gaseoso para transporte a partir de biomasa, algunas propiedades del biodiésel son prácticamente las mismas que las del gasóleo (gasoil) de automoción en cuanto a densidad y número de cetano. Además, presenta un punto de inflamación superior. Por todo ello, el biodiésel puede mezdarse con el gasóleo para su uso en motores e incluso sustituirlo totalmente si se adaptan éstos convenientemente. Por otra parte, la definición de biodiésel propuesta por las especificaciones ASTM (American Society for Testing and Material Standard, asociación intemacional de normativa de calidad) lo describe como ésteres mono alquílicos de ácidos grasos de cadena larga derivados de lípidos renovables tales como aceites vegetales o grasas de animales, y que se emplean en motores de ignición de compresión. Según Brown, et al (2004), para llevar a cabo la producción de biodiesel es necesario contar con la presencia de catalizadores para que ésta reacción ocurra y sea posible desde un punto de vista cinético, ya que éste proporciona una secuencia altemativa de la reacción con una menor energía de activación. Estos catalizadores, en el caso de la transesterificación (reacción mediante la cual se obtiene el biodiesel), pueden ser ácidos homogéneos, ácidos heterogéneos, básicos homogéneos 0 enzimáticos, siendo los catalizadores básicos los que se utilizan a nivel industrial en la transesterificación ya que actúan mucho más rápido y además permiten operar en condiciones moderadas. Siendo la Catálisis Homogénea la via por la cual, se tiene un acceso más fácil al mecanismo de reacción y por consecuencia se puede determinar mejor el proceso catalítico que se lleva acabo. Por otra parte, la ausencia de efectos de envenenamiento es una ventaja de la catálisis homogénea, además el impulso que han dado sus complejos organometálicos ha dado pie para su aplicación en la industria.

Por otro lado la sensibilización en cuanto los daños que se producen al medio Ambiente, los Procesos de Producción Industrial, y por ende la rápida Industrialización y tecnificación mundial, no están lejos de ser involucrados en los contenidos curriculares de las Instituciones Educativas, en especial porque es allí en donde se crea el puente entre los conceptos cientúficos y la vida real, en donde se materializa las teorías y las leyes, y en donde los estudiantes adquieren conocimientos significativos, ahora bien, la construcción de dichos conceptos científicos, mediante la relaciones entre los procesos de industrialización y las técnicas Educativas, así como los desafíos y retos ante las nuevas líneas de investigación en didácticas especiales, como es el caso de química, obligan a que los estudiantes se vinaulen con las nuevas tecnológias de la información y Comunicación, para 
que se desarrollen habilidades cognitivas y se consiga potenciar el pensamiento critico y reflexivo en los estudiantes.

\section{Tics en la enseñanza de la química}

La sociedad actualmente presenta grandes retos no solo en el ámbito ambiental sino también a nivel educativo, ya que las Tecnologías de Información y Comunicación (TIC) han cambiado la manera de relacionarnos con otros, revolucionado muchos campos de la actividad humana Galvis, (2004), imponiendo a los profesionales de la educación el reto de asumirlas y desarrollarlas con miras a que se constituyan en mecanismos efectivos de movilización cultural y contribuyan al desarrollo humano. En un mundo que evoluciona constantemente, la oferta de recursos informáticos y tecnológicos va aumentando cada vez más, lo cual se hace más evidente como la aparición de nuevos y mas sofisticados computadores, teléfonos móviles, conexiones a internet, chats, plataformas, entre otros, lo cual genera, dudas de los beneficios que aportan estos avances en el ámbito educativo, pero, sin embargo es necesario plantearlos en el seno de una perspectiva global, valorando las causas y, sobre todo, las necesidades reales de su adopción Galvis, (2004).

El comportamiento de las sociedades y los individuos ante el cambio de mentalidad esta relacionado con la educación y con el impacto de ésta en la actitud de las personas. Si los ciudadanos del "actual futuro" no tienen una sólida actitud hacia el cambio, tendrán graves problemas no sólo desde el punto de vista de los conocimientos útiles sino de las habilidades intelectuales y técnicas para enfrentar las exigencias del desarrollo científico y teconológico Iriarte, (2006), con la implementación de las TIC (teanologías de la información y la comunicación) en el ámbito educativo.

Con base en lo mencionado anteriormente, se trata de poner de manifiesto cómo la química siendo una ciencia básica que tiene multitud de relaciones con los problemas que atañen al planeta y, por tanto, a los seres humanos, debe aprovechar la potencialidad que pueden o presentan la implementación de las TICS, para orientar a los estudiantes hacia las posibles soluciones, que se concretan en un desarrollo sostenible, que comprometa a cada uno de los estudiantes con un desarrollo científico, tecnológico, social y económico responsable y solidario con el planeta tiena ( Santana et al,2005) debido a las grandes problemáticas que esta presentando actualmente y del aul no se puede ser indiferente ya que sus efectos están directamente relacionados con cada habitante de la tiena.

Entonces, es preciso encontrar los usos pedagógicos adecuados, las mejores formas de insertar en el currículo las nuevas tecnologías de la información y la comunicación, diseñar los procesos que conduzcan a esa incorporación exitosa de éstas en la educación, y especialmente en la química, pero, además que la implementación de éstas herramientas, como lo son las TICS, ayuden a las estudiantes a vivir "experiencias directas interactuando con el objeto de conocimiento, con modelos del mismo, con personas que tienen distintas perspectivas sobre el mismo, así como a construir y expresar sus propios modelos mentales"(Galvis, 2004).

\section{Descripción del problema}

El desafío actualmente en el ámbito educativo está en potencializar habilidades en las nuevas generaciones para que puedan desarrollar pensamiento crítico y reflexivo frente a las problemáticas que surgen en la cotidianidad para que ellos actúen de manera propositiva en la construcción y mejoramiento de su entorno, además de responder a los retos que se presentan, participar activamente en la sociedad del conocimiento, y contribuir al desarrollo sostenible del medio ambiente, todo esto se puede conseguir con base en la formulación de preguntas, interpretación de resultados, y construcción de modelos argumentativos en las que se debe contar con la participación de profesores y estudiantes en general para que los resultados sean óptimos. 
Desde esta perspectiva, es del interés de esta investigación cuestionarse alrededor de la siguiente problemática, que es el eje de trabajo en el proceso de construcción del proyecto:

¿Es viable llevar al aula aspectos de interés Social, Ambiental y Económico, como la producción de Combustibles Altemativos a partir de Desechos Orgánicos y convertirlos en herramientas de Enseñabilidad, que permitan el desarrollo de habilidades cognoscitivas para fomentar el pensamiento crítico y reflexivo en los estudiantes?

A partir se esto se plantea como objetivo principal Abordar el estudio de ciertos Aspectos de interés Social, Económico, y Ambiental, como la producción de combustibles Altemativos a partir de Desechos Orgánicos, y asociarlos al trabajo docente y educativo, convirtiéndolos en Herramientas de Enseñabilidad, que permitan el desarrollo de habilidades en los estudiantes, con el fin de mejorar su pensamiento crítico y reflexivo.

\section{Metodología}

Con el fin de conseguir llevar a cabo los objetivos propuestos en la investigación, se plantean dos etapas generales, que giran en tomo al enfoque pedagógico y didáctico:

\section{Descripción del enfoque pedagógico y didáctico}

Constituye el referente Metodológico, se hace con base a una revisión teórica, con respecto a las concepciones de Competencias y las estrategias que se han tenido en cuenta para su desarrollo. Luego de la revisión teórica se construyen los instrumentos de ideas previas, los cuales buscan identificar el nivel de habilidades de pensamiento cuítico y científico en los estudiantes.

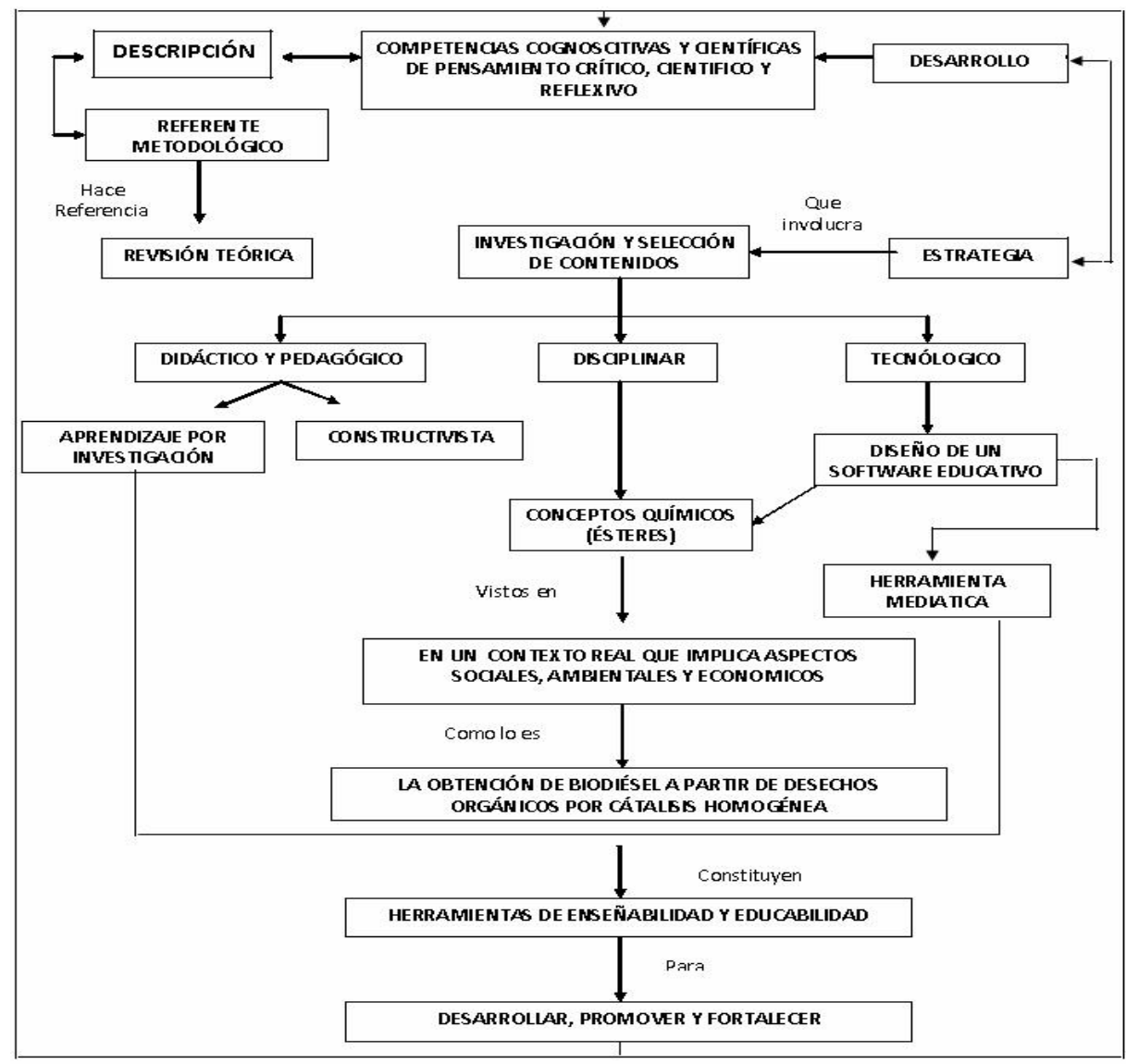


Gráfico 1. Proceso Metodológico Cualitativo

El instrumento cualitativo contribuye de manera práctica al desarrollo de la escuela, el aula, la profesión y la formación de profesionales, razón por la que constituye un fenómeno mundial aplicado a la educación, además por que no solo implica registrar lo que ocurre descriptivamente de forma muy detallada, sino también por que permite recopilar y analizar reacciones e impresiones entomo a lo que ocurre.

Desarrollo de la Estrategia

En donde se investigan y seleccionan los contenidos pedagógicos y didácticos a utilizar, así como el contenido disciplinar y la herramienta mediáticas.

\section{Conclusiones}

El proyecto de investigación se propone como una estrategia de enseñabilidad, que busca desarrollar, promover y fortalecer las habilidades cognoscitivas y de pensamiento critico y reflexivo con respecto a ciertos conceptos científicos en especial los químicos, desde de la relación existente entre un contexto con aspectos de interés económico, social y ambiental como la obtención de Biodiésel a partir de Desechos orgánicos y de herramientas mediáticas y tecnológicas como el diseño e implementación de un software.

\section{Bibliografía}

Cárdenas, F. Sarmiento, R. (2000). Desarrollo y Evaluación de los procesos de razonamiento complejo en ciencias. Revista TEム 3.

Gallego, R. y Pérez, R. (1998). Aprendibilidad - Educabilidad - Enseñabilidad: Una discusión, Revista Colombiana de Educación

Gallego, R. y Pérez, R. (1999). La Construcción de Competencias, Una Intencionalidad Curriaular: Bogotá, Colombia: Paradigma Editorial.

Galvis, A. (2004). Oportunidades educativas de las TIC. Meta recursos, Soluciones Learning Innovadoras, D. Ed. Concord, MA, 1-6.

Iriarte, F. (2006). Incorporación de TICS en las actividades cotidianas del aula: una experiencia en escuela de provincia. Revista del Instituto de Estudios en Educación Universidad del Norte. № 7, Diciembre, ISSN 1657-2416.

Salcedo, L, Villareal, M., Zapata, P., Colmenares, E., García, M., Moreno, S. (2008). Tecnologías de la Información y la Comunicación en educación en Química. Bogotá, Colombia: Universidad Pedagógica Nacional y Colciencias.

Santana, A., Navarro, F., Fernández, E., González, V., Domínguez, J. (2005). Aprender química para un futuro sostenible. Aspectos CTSA en la química de $2^{\circ}$ de bachillerato utilizando las TIC. Grupo Lentiscal de Didáctica de la Física y Química. España. 